\title{
Extracorporeal life support in adult patients with out-of-hospital cardiac arrest
}

\author{
Jia Hao $\underline{\text { Lim }}^{1}$, MBBS, MMed(EM), Mathew Jose $\underline{\text { Chakaramakkil}}{ }^{2}$, MBBS, FRCS, Boon Kiat Kenneth $\underline{\operatorname{Tan}}^{1}$, MBBS, MMed(EM)
}

\begin{abstract}
The use of extracorporeal life support in cardiopulmonary resuscitation (CPR) of adult patients experiencing out-of-hospital cardiac arrest by the application of veno-arterial extracorporeal membrane oxygenation (ECMO) during cardiac arrest has been increasing over the past decade. This can be attributed to the encouraging results of extracorporeal CPR (ECPR) in multiple observational studies. To date, only one randomised controlled trial has compared ECPR to conventional advanced life support measures. Patient selection is crucial for the success of ECPR programmes. A rapid and organised approach is required for resuscitation, i.e. cannula insertion with ECMO pump initiation in combination with other aspects of post-cardiac arrest care such as targeted temperature management and early coronary reperfusion. The provision of an ECPR service can be costly, resource intensive and technically challenging, as limited studies have reported on its cost-effectiveness.
\end{abstract}

Keywords: cardiopulmonary resuscitation, extracorporeal life support, extracorporeal membrane oxygenation, out-of-hospital cardiac arrest

\section{INTRODUCTION}

Out-of-hospital cardiac arrest (OHCA) is associated with poor survival rates worldwide.(1) In Singapore, the rising incidence of OHCA owing to its ageing population is a health concern. Historically, only $2.5 \%$ chance of survival from OHCA has been reported in Singapore. ${ }^{(2)}$ However, the implementation of a dispatch-assisted cardiopulmonary resuscitation (CPR) programme in Singapore, as well as improved public education, has led to an increase in bystander CPR rates, resulting in an increased survival rate of around $5 \%$. $^{(3)}$

Patients with OHCA with pre-hospital cardiac arrest rhythms of ventricular fibrillation (VF) or ventricular tachycardia (VT) are more likely to survive compared with patients with non-shockable rhythms. ${ }^{(2,4)}$ Unfortunately, a significant number of these patients remain in refractory cardiac arrest despite advanced life support (ALS) interventions and do not achieve return of spontaneous circulation (ROSC).

Extracorporeal life support (ECLS) in cardiopulmonary resuscitation (ECPR) is the implantation of veno-arterial extracorporeal membrane oxygenation (VA ECMO) in patients with refractory cardiac arrest when conventional CPR (CCPR) measures fail to achieve sustained ROSC. ${ }^{(5)} \mathrm{VA} \mathrm{ECMO} \mathrm{is} \mathrm{a}$ procedure where patients are connected to an extracorporeal circuit via large cannulas inserted into their central venous system (typically via the femoral vein into the inferior vena cava) and the arterial system (via the femoral artery). The extracorporeal circuit, which comprises the centrifugal blood pump and membrane oxygenator, aims to support circulation and organ perfusion, as well as provide gas exchange by pumping blood from the circulation out through the venous cannula, passing it through the extracorporeal circuit and returning it via the arterial cannula. This provides time for the patients to receive urgent interventions that might help bridge the patients to circulatory recovery. ECPR for patients with OHCA often involves the delivery of bundled care, which includes targeted temperature management (TTM) and early definitive treatment that might help improve native circulation (percutaneous coronary interventions or thrombus removal for massive pulmonary embolism). It can also support patients awaiting recovery from myocardial stunning, myocarditis or cardiotoxic agents. ${ }^{(6)}$

The use of ECPR for OHCA has been increasing over the past decade ${ }^{(7)}$ despite limited high-quality data from randomised controlled studies (only one such study published to date). ${ }^{(8)}$ Based on data from the Extracorporeal Life Support Organization (ELSO) Registry, the rising number of ECMO centres $^{(9)}$ and observational studies reporting favourable results (higher survival rates compared with those observed with CCPR) might account for this trend. ${ }^{(10-17)}$

\section{ECPR VERSUS CCPR IN ADULT OHCA}

Patients who experience in-hospital cardiac arrest (IHCA) show an approximately $30 \%$ chance of survival after receiving $\mathrm{ECPR},{ }^{(9,16)}$ with considerably shorter times from cardiac arrest to ECMO. ${ }^{(16,18)}$ Compared with IHCA, patients with OHCA face different circumstances and challenges owing to the occurrence of cardiac arrest in the community. For example, the time from experiencing cardiac arrest to commencing CPR may be longer and the quality of CPR may be poorer, as early bystander response may not come from trained providers. The longer transport times to a hospital where they subsequently receive ECPR (if they fit the strict patient selection criteria) can also negatively affect outcomes and survival due to the longer 'low-flow' time before they are started on ECMO. ${ }^{(19)}$ Despite these significant obstacles and challenges to ECPR for patients with OHCA, small single-centre studies ${ }^{(10)}$ and prospective observational cohort studies have reported encouraging results, 
which suggest that ECPR could yield better survival outcomes than CCPR does for OHCA.

In a large, multicentre prospective observational study by Sakamoto et al, ECPR was compared to CCPR for patients who experienced OHCA. ${ }^{(20)}$ The study involved 46 hospitals, all of which were capable of performing post-cardiac arrest interventions such as percutaneous coronary interventions ( $\mathrm{PCl})$ and TTM. A total of 26 selected hospitals capable of performing ECPR were assigned to perform ECPR for OHCA, and 20 hospitals were assigned to perform CCPR as per their normal resuscitation strategies. A total of 454 adult patients who experienced OHCA with VF or VT as their initial rhythm were enrolled in the study and the groups were compared, with the primary endpoints being favourable neurological outcomes at one and six months after cardiac arrest. The intention-to-treat analysis demonstrated better neurological outcomes at one month after cardiac arrest in the ECPR group compared to the CCPR group (12.3 vs. $1.5 \%$, $\mathrm{p}<0.0001)$ and similar results at six months $(11.2 \%$ vs. $2.6 \%$, $p=0.001)$. However, there were several limitations to this study. Owing to the dichotomous assignment of hospitals to a single modality of CPR (ECPR vs. CCPR), the study risked introducing biases attributable to differences in the quality of care between the ECPR and CCPR groups. Health systems familiar with ECPR might have better facilities suited for other endovascular interventions such as $\mathrm{PCl}$, intra-aortic balloon pump (IABP) insertion and therapeutic hypothermia. It should also be noted that, while most (91.5\%) of the patients with ECPR received interventions such as therapeutic hypothermia, only half $(54.1 \%)$ of the patients with CCPR received this intervention. Similarly, the rate of IABP insertion was $92.7 \%$ in the ECPR group compared to $62.2 \%$ in the CCPR group. These post-cardiac arrest bundled interventions also likely contributed to the improved neurological outcomes in the ECPR group. The difference in post-cardiac arrest intervention rates in the ECPR group compared with the CCPR group might not be due to deviations in post-cardiac arrest protocols or a difference in the quality of treatment for patients with CCPR. Patients with CCPR who were too unstable to tolerate such interventions despite vasopressors or inotropes could not be started on hypothermia or undergo further invasive interventions, compared with patients with ECPR who were more likely to receive such interventions when haemodynamically supported with ECMO.

Similar promising results were reported in a smaller propensity-matched observational study by Maekawa et al, where ECPR was compared with CCPR in adult patients with OHCA. ${ }^{(21)}$ In this study, 162 patients who met the study inclusion criteria were enrolled (53 ECPR vs. 109 CCPR) and evaluated for the endpoint of a positive neurological outcome of CPC 1 or 2 at three months after cardiac arrest. Improved outcomes were observed in the ECPR group compared with the CCPR group $(29.2 \%$ vs. $8.3 \%, \log$-rank $p=0.018$ ). Of note, patients with ECPR were much more likely to receive post-cardiac arrest interventions such as therapeutic hypothermia $(49.1 \%$ vs. $6.4 \%)$, IABP $(50.9 \%$ vs. $9.2 \%)$ and $\mathrm{PCl}(39.6 \%$ vs. $5.5 \%)$.

By contrast, Bougouin et al published an analysis of a large registry of patients with OHCA in Paris, comparing ECPR with CCPR. ${ }^{(22)}$
In this analysis of 525 patients treated with ECPR and 12,666 patients treated with CCPR, the primary outcome of survival to hospital discharge was assessed. There was no difference in survival to discharge between the two groups (ECPR $8.4 \%$ vs. CCPR $8.6 \%, p=0.91$ ). In the adjusted multivariable analysis, ECPR was not significantly associated with survival (odds ratio [OR] 1.3, 95\% confidence interval [CI] 0.8-2.1; $\mathrm{p}=0.24$ ). However, in patients who did receive ECPR, three factors were found to be independently associated with greater survival: initial shockable rhythm (OR 3.9, 95\% Cl 1.5-10.3; $\mathrm{p}=0.005)$, ROSC during initial resuscitation (OR 2.3, 95\% Cl 1.1-4.7, $\mathrm{p}=0.03$ ) and pre-hospital ECMO (OR 2.9, 95\% Cl 1.5-5.9; $p=0.002)$. It should be noted that there were no strict inclusion criteria owing to the observational analysis of unselected multicentre registry data and that ECPR was initiated at the discretion of the treating physicians, which risked the introduction of selection bias.

Several systematic reviews have compared ECPR with CCPR and suggested that ECPR might be associated with improved survival to discharge or neurological outcomes. ${ }^{(23-27)}$ However, all systematic reviews have rated the quality of evidence as low, with risk of bias. A recent systematic review by Holmberg et al for the International Liaison Committee on Resuscitation's Advanced Life Support and Paediatric Task Forces demonstrated mixed results. Of the 12 studies identified to assess survival to discharge in adult $\mathrm{OHCA}$, seven showed higher odds of survival with ECPR and the remaining five showed lower odds of survival compared with CCPR. ${ }^{(28)} \mathrm{A}$ meta-analysis was not performed owing to high study heterogeneity and serious risk of bias. A meta-analysis of ECPR vs. CCPR studies was performed by Kim et al, focusing on the endpoints of survival to discharge and good neurological outcomes at 3-6 months. ${ }^{(29)}$ In total, ten studies from the Asian health systems of South Korea, Japan and Taiwan were included in the meta-analysis. While this meta-analysis demonstrated improved survival and neurological outcomes at 3-6 months in the ECPR group compared with the CCPR group in the overall analyses, subgroup analyses stratified by the location of cardiac arrest (OHCA vs. IHCA) did not show any clear beneficial effects of ECPR in patients with OHCA for survival to discharge (relative risk 1.45, 95\% $\mathrm{Cl}$ 0.41-5.16).

The 2019 International Consensus on Cardiopulmonary Resuscitation and Emergency Cardiovascular Care Science with Treatment Recommendations weakly recommends that ECPR "can be considered as a rescue therapy for selected patients with cardiac arrest when CCPR is failing in settings where it can be implemented". ${ }^{(30)}$ When weighed against the extremely high mortality of refractory cardiac arrest when it is managed with standard ALS measures, the potential for benefit from ECPR still remains despite the overall low certainty of supporting evidence. Further randomised controlled studies are warranted to clarify this issue.

The first of such randomised controlled studies by Yannopoulos et al, the recently published ARREST trial, compared ECPR against standard ALS measures for the management of adult patients with OHCA and refractory VF. ${ }^{(8)}$ Refractory VF in this study's inclusion criteria was: OHCA with presumed cardiac 
aetiology; first presenting rhythm VF; age 18-75 years; having received at least three shocks without sustained ROSC; and having received amiodarone. Patients with do-not-resuscitate orders, traumatic injuries or burns injuries; those who had ever experienced drowning; those with active or uncontrolled bleeding; and those with advanced malignancies were excluded. In this Phase 2, open-label, single-centre study, 30 patients were randomised to receive either early ECMO-facilitated resuscitation or standard ALS treatment. In the ECPR group, patients gained immediate access to the cardiac catheterisation laboratory upon hospital arrival, where resuscitation was continued. If a patient did not meet pre-established resuscitation termination criteria, ECMO support was initiated, followed by immediate coronary revascularisation. The control group remained in the emergency department (ED) under the management of emergency physicians, where standard ALS measures were continued. If ROSC was achieved, the patient was transferred to the cardiac catheterisation laboratory for further cardiac care and angiography, as required. Post-resuscitation care followed local standards of care, which included 24 hours of therapeutic hypothermia and continuous electroencephalogram monitoring. Neuro-prognostication was performed at least 72 hours after cardiac arrest. The primary outcome of survival to hospital discharge was studied and analysed using the Bayesian model in 29 patients (as one patient withdrew consent after randomisation). The primary outcome was observed in six out of 14 patients in the ECPR group (43\%, 95\% credible interval 21.3-67.7), compared with one out of 15 patients in the standard ALS group (7\%, 95\% credible interval 1.6-30.2). The ECMO group also fared better in the secondary outcome of cumulative survival (hazard ratio $0.16,95 \% \mathrm{Cl} 0.06-0.41$; logrank test $\mathrm{p}<0.0001)$. Modified Rankin scores in the ECMO group improved over six months, with time and physical rehabilitation. All six patients who received ECPR remained alive at six months, scoring CPC 1 to 2. Statistical analysis between the ECMO and the ALS groups was not possible at three and six months, as one patient in the ALS group died before three months.

It should be noted that the positive outcomes of the ARREST trial can also possibly be attributed to the existence of well-developed protocols in an efficient, multidisciplinary and community-wide systems approach in the management of refractory OHCA secondary to VF/VT that involves close collaborations between interventional cardiology, EDs and out-ofhospital emergency medical services (EMS) providers, including mobile ECMO teams. ${ }^{(31)}$

\section{PATIENT SELECTION AND INDICATIONS}

There is no consensus on the accepted indications for ECPR to date. The common inclusion criteria used in most ECPR studies on adult patients ${ }^{(32,33)}$ are: (a) age between 18 and 65-75 years; (b) cardiac arrest with bystander CPR; (c) an initial shockable cardiac arrest rhythm; (d) $<5$ minutes of 'no-flow' time (time from cardiac arrest to CPR); (e) $<60$ minutes of anticipated 'low-flow' time (time interval to place patient on ECMO); (f) end-tidal $\mathrm{CO}_{2}$ readings $>10 \mathrm{mmHg}$; and (g) 'signs of life' (spontaneous movements, breathing, gasping and pupillary reflexes) independent of the cardiac rhythm. However, these criteria have not been prospectively validated.

Common contraindications to ECPR are patients with do-notresuscitate orders, traumatic cardiac arrest, severe brain injury, terminal malignancies, active or uncontrolled bleeding, chronic end-organ dysfunction, suspected aortic dissections and aortic valve incompetency.

The recent Interim Guideline Consensus Statement from the ELSO recommended that 'locally agreed inclusion criteria' be formulated to help clinicians ensure appropriate allocation of resources to patients with the highest chances of survival after cardiac arrest. ${ }^{(32)}$

\section{COMPLICATIONS OF ECPR}

It is important to be familiar with the complications of ECMO, as they can lead to negative outcomes and death. Bleeding is the commonest complication associated with ECPR, ${ }^{(34)}$ owing to anticoagulation-associated bleeding into the cerebral or gastrointestinal system, large-vessel bleeding at the cannulation sites, as well as CPR-associated complications such as fractures, haemothoraxes and pulmonary haemorrhages. ${ }^{(35)}$ Other common complications related to ECPR are unsuccessful cannulation, limb ischaemia and infection. ${ }^{(36)}$

$\mathrm{ECMO}$ is also associated with high incidences of neurological complications, such as anoxic brain injury, ischaemic strokes, cerebral haemorrhage, seizures, cerebral oedema and brain death. The mechanisms of such injuries are complex, and can be due to the cardiac arrest as well as from ECMO support. A systematic review and meta-analysis by Migdady et al reported a frequency of hypoxic-ischaemic brain injury at $23 \%(95 \% \mathrm{Cl} 0.14 \%-0.32 \%)$, ischaemic stroke at $6 \%(95 \% \mathrm{Cl} 0.02 \%-0.11 \%)$ and intracerebral haemorrhage at $4 \%(95 \% \mathrm{Cl} 0.01 \%-0.1 \%){ }^{(37)}$

\section{TIMING OF ECPR}

The emplacing of a cardiac arrest victim onto a VA ECMO circuit with ongoing ALS measures is a challenging task. This is a complex intervention that requires strong teamwork and communication, well-defined roles and trained healthcare providers. ${ }^{(30)}$ As most EDs that manage patients with OHCA rely on a separate ECPR response team to perform the $\mathrm{ECMO}$ implantation while the ED team is delivering ALS, it should be recognised that there ought to be a timely transition from the initial ALS measures to ECPR. There has been no consensus regarding the optimal time to commence ECPR. Kim et al suggested a time of 21 minutes as the decision point to switch from CCPR to ECPR. ${ }^{(38)}$ Cardarelli et al reported that patients who receive more than 30 minutes of CPR before initiation of ECMO are more likely to die than those who receive less than 30 minutes of CPR. ${ }^{(39)}$ However, ECPR should not be commenced too early, when early ROSC from CCPR can still potentially occur. The costs and complications of ECPR that can be inflicted on a patient who might not have needed it in the first place can outweigh any of the benefits it can confer; yet, if commenced too late, the prolonged hypoperfusion can result in poorer survival owing to increased risk of anoxic and reperfusion injuries to the brain and end-organs. 
Observational studies have demonstrated improved survival after ECPR among patients with a shorter interval of 'low-flow', which is the period between the time of cardiac arrest and the establishment of ECMO flow. The recent Interim Guideline Consensus Statement from the ELSO recommends that the goal of ECPR is to establish adequate ECMO flow within 60 minutes of cardiac arrest. ${ }^{(32)}$ This is a significant logistical challenge, as the time taken to deploy ECMO may differ between health systems and institutions owing to variations in infrastructure, staffing, training, pre-hospital transport times and EMS protocols (e.g. 'stay and treat' vs. 'scoop and run'). In order to reduce the low-flow times further, some health systems have adopted pre-hospital ECMO with highly specialised mobile ECLS teams that will assess and make the decision to commence ECPR at the scene of OHCA if the patient fits the ECPR criteria, before transporting the patient back. ${ }^{(31,40,41)}$ The time to make a decision for ECPR might have to take into account the 'lead time' that a trained ECMO team requires to cannulate and emplace a patient with cardiac arrest onto an ECMO circuit.

\section{COST-EFFECTIVENESS AND RESOURCE MANAGEMENT}

An effective ECPR service requires significant investment in staffing, training, intensive care unit (ICU) beds and equipment for the delivery of ECMO, as well as the post-resuscitation care to be delivered in the cardiac catheterisation laboratory and the ICU. To date, limited studies regarding the cost-effectiveness of ECPR for adult patients with OHCA have been reported. As ECMO technology continues to evolve, and as costs between healthcare systems can vary significantly between studies, geography and time, it is difficult to perform systematic reviews and metaanalyses on these highly heterogeneous studies. ${ }^{(42)}$

Recent studies from Japan and Australia that included adults with OHCA who received ECPR suggest that this technique might be highly cost-effective for the treatment of patients with OHCA who have refractory VT/VF. ${ }^{(43-45)}$ However, these studies also acknowledge that there is limited long-term outcome data regarding the prognosis of these cardiac arrest survivors after ECPR and that it was difficult to account for long-term healthcare costs in their analyses. In addition, data was insufficient to account for the costs incurred in the acute phase of resuscitation; hence, some costs might have been missed or inaccurate, thus overestimating the cost-effectiveness of ECPR. To date, no study has assessed the cost-effectiveness of implementing a pre-hospital ECPR programme.

Evidence suggests that experience in delivering ECMO is associated with improved patient outcomes. Barbaro et al found a positive association between high volume of ECMO referral centres and survival outcomes in adults and neonates receiving ECMO. ${ }^{(46)}$ For local centres that do not have specialist ECMO services or are inexperienced in ECMO or ECPR, investment in the development of an ECPR service, where patients bear the risk of suboptimal results or complications, might not be justified. The creation of regional referral systems to tertiary ECMO specialist centres that have the ability to deploy mobile ECMO teams to initiate ECMO and transport patients back to the centre can address this gap.

However, in the context of ECPR for OHCA, where time to $\mathrm{ECMO}$ is critical, this raises questions on how effective these mobile ECMO teams can be in delivering positive outcomes when it is suggested that ECMO should be provided within 60 minutes of cardiac arrest. A public healthcare system where selected patients with OHCA in geographical areas in close proximity to tertiary ECMO referral centres are more likely to have access to early ECPR, as opposed to those who are distant, challenges the ideals of distributive justice. ${ }^{(47)}$ Rather than channelling valuable public resources into the development of a pre-hospital ECPR programme or creating ECMO centres out of every hospital, prioritising the development of standardised national post-cardiac arrest bundled care protocols for all cardiac arrest survivors or bystander CPR campaigns might be more cost-effective in delivering positive cardiac arrest survival outcomes to the population as a whole.

The potential benefits of ECPR in OHCA should always be carefully balanced against the complications, cost and medical futility; this highlights the need for stringent patient selection to achieve positive outcomes. Poor patient selection will result in wasted resources, which could otherwise be used for other patients.

\section{CONCLUSION}

An effective ECPR programme with bundled post-cardiac arrest care (TTM and early cardiac interventions with high-quality ICU care with neurological monitoring) for refractory OHCA may improve outcomes in highly selected patients. Timely implementation of ECMO in patients with OHCA by well-drilled teams, combined with strong, multidisciplinary post-cardiac arrest care, might allow such patients to receive further advanced targeted therapies in the ICU as well as cardiac catheterisation laboratories. Further randomised multicentre studies to evaluate ECPR and its role in bridging patients to post-cardiac arrest management can lead to increased understanding and efforts for health systems to improve the delivery of this highly complex intervention to patients with cardiac arrest.

\section{REFERENCES}

1. Berdowski J, Berg RA, Tijssen JG, Koster RW. Global incidences of out-of-hospital cardiac arrest and survival rates: systematic review of 67 prospective studies. Resuscitation 2010; 81:1479-87.

2. Ong MEH, Sang DS, De Souza N, et al. Outcomes for out-of-hospital cardiac arrests across 7 countries in Asia: The Pan Asian Resuscitation Outcomes Study (PAROS). Resuscitation 2015; 96:100-8. Erratum in: Resuscitation 2016; 98:125-6.

3. White AE, Poh JS, Pek PP, et al. Singapore out-of-hospital cardiac arrest registry report 2011-2018. November 2020. In: Unit for Pre-hospital Emergency Care, Singapore. Available at: https://www.myheart.org.sg/wp-content/uploads/2021/01/ Singapore-OHCA-Data-Report-2011-2018.pdf. Accessed May 1, 2021.

4. Neumar RW, Nolan JP, Adrie C, et al. Post-cardiac arrest syndrome: epidemiology, pathophysiology, treatment, and prognostication. A consensus statement from the International Liaison Committee on Resuscitation (American Heart Association, Australian and New Zealand Council on Resuscitation, European Resuscitation Council, Heart and Stroke Foundation of Canada, InterAmerican Heart Foundation, Resuscitation Council of Asia, and the Resuscitation Council of Southern Africa); the American Heart Association Emergency Cardiovascular Care Committee; the Council on Cardiovascular Surgery and Anesthesia; the Council on Cardiopulmonary, Perioperative, and Critical Care; the Council on Clinical Cardiology; and the Stroke Council. Circulation 2008; 118:2452-83. 
5. Inoue A, Hifumi T, Sakamoto T, Kuroda Y. Extracorporeal cardiopulmonary resuscitation for out-of-hospital cardiac arrest in adult patients. J Am Heart Assoc 2020; 9:e015291.

6. Swol J, Belohlávek J, Brodie D, et al. Extracorporeal life support in the emergency department: a narrative review for the emergency physician. Resuscitation 2018; 133:108-17.

7. Richardson AS, Schmidt $M$, Bailey $M$, et al. ECMO Cardio-Pulmonary Resuscitation (ECPR), trends in survival from an international multicentre cohort study over 12-years. Resuscitation 2017; 112:34-40. Comment in: J Thorac Dis 2017; 9:1415-9.

8. Yannopoulos D, Bartos J, Raveendran G, et al. Advanced reperfusion strategies for patients with out-of-hospital cardiac arrest and refractory ventricula fibrillation (ARREST): a phase 2, single centre, open-label, randomised controlled trial. Lancet 2020; 396:1807-16.

9. Extracorporeal Life Support Organization. ECLS registry report international summary. Available at: https://www.elso.org/registry/statistics/ InternationalSummary.aspx. Accessed April 1, 2021.

10. Stub D, Bernard S, Pellegrino V, et al. Refractory cardiac arrest treated with mechanical CPR, hypothermia, ECMO and early reperfusion (the CHEER trial). Resuscitation 2015; 86:88-94.

11. Dennis M, Buscher H, Gattas D, et al; Sydney ECMO Research Interest Group. Prospective observational study of mechanical cardiopulmonary resuscitation extracorporeal membrane oxygenation and early reperfusion for refractory cardiac arrest in Sydney: the 2CHEER study. Crit Care Resusc 2020; 22:26-34.

12. Chen YS, Chao A, Yu HY, et al. Analysis and results of prolonged resuscitation in cardiac arrest patients rescued by extracorporeal membrane oxygenation. J Am Coll Cardiol 2003; 41:197-203.

13. Chen YS, Lin JW, Yu HY, et al. Cardiopulmonary resuscitation with assisted extracorporeal life-support versus conventional cardiopulmonary resuscitation in adults with in-hospital cardiac arrest: an observational study and propensity analysis. Lancet 2008; 372:554-61.

14. Lazzeri C, Sori A, Bernardo P, et al. In-hospital refractory cardiac arrest treated with extracorporeal membrane oxygenation: a tertiary single center experience. Acute Card Care 2013; 15:47-51.

15. Shin TG, Choi JH, Jo IJ, et al. Extracorporeal cardiopulmonary resuscitation in patients with inhospital cardiac arrest: a comparison with conventional cardiopulmonary resuscitation. Crit Care Med 2011; 39:1-7.

16. Kagawa E, Inoue I, Kawagoe T, et al. Assessment of outcomes and differences between in- and out-of-hospital cardiac arrest patients treated with cardiopulmonary resuscitation using extracorporeal life support. Resuscitation 2010; 81:968-73.

17. Le Guen M, Nicolas-Robin A, Carreira S, et al. Extracorporeal life support following out-of-hospital refractory cardiac arrest. Crit Care 2011; 15:R29.

18. Lunz D, Calabrò L, Belliato M, et al. Extracorporeal membrane oxygenation for refractory cardiac arrest: a retrospective multicenter study. Intensive Care Med 2020; 46:973-82.

19. Wengenmayer T, Rombach S, Ramshorn F, et al. Influence of low-flow time on survival after extracorporeal cardiopulmonary resuscitation (eCPR). Crit Care $2017 ; 21: 157$

20. Sakamoto T, Morimura N, Nagao K, et al; SAVE-J Study Group. Extracorporeal cardiopulmonary resuscitation versus conventional cardiopulmonary resuscitation in adults with out-of-hospital cardiac arrest: a prospective observational study. Resuscitation 2014; 85:762-8. Comment in: Resuscitation 2014; 85:709-10.

21. Maekawa K, Tanno K, Hase M, Mori K, Asai Y. Extracorporeal cardiopulmonary resuscitation for patients with out-of-hospital cardiac arrest of cardiac origin: a propensity-matched study and predictor analysis. Crit Care Med 2013; 41:118696. Comment in: Crit Care Med 2013; 41:1365-6.

22. Bougouin W, Dumas F, Lamhaut L, et al; Sudden Death Expertise Center investigators. Extracorporeal cardiopulmonary resuscitation in out-of-hospital cardiac arrest: a registry study. Eur Heart J 2020; 41:1961-71.

23. Beyea MM, Tillmann BW, lansavichene AE, et al. Neurologic outcomes after extracorporeal membrane oxygenation assisted CPR for resuscitation of outof-hospital cardiac arrest patients: a systematic review. Resuscitation 2018; 130:146-58

24. Ortega-Deballon I, Hornby L, Shemie SD, Bhanji F, Guadagno E. Extracorporeal resuscitation for refractory out-of-hospital cardiac arrest in adults: a systematic review of international practices and outcomes. Resuscitation 2016; 101:12-20.

25. Debaty G, Babaz V, Durand M, et al. Prognostic factors for extracorporeal cardiopulmonary resuscitation recipients following out-of-hospital refractory cardiac arrest. A systematic review and meta-analysis. Resuscitation 2017; 112:1-10
26. Twohig CJ, Singer B, Grier G, Finney SJ. A systematic literature review and meta-analysis of the effectiveness of extracorporeal-CPR versus conventionalCPR for adult patients in cardiac arrest. J Intensive Care Soc 2019; 20:347-57.

27. Gravesteijn BY, Schluep M, Disli M, et al. Neurological outcome after extracorporeal cardiopulmonary resuscitation for in-hospital cardiac arrest: a systematic review and meta-analysis. Crit Care 2020; 24:505.

28. Holmberg M, Geri G, Wiberg S, et al; International Liaison Committee on Resuscitation's (ILCOR) Advanced Life Support and Pediatric Task Forces. Extracorporeal cardiopulmonary resuscitation for cardiac arrest: a systematic review. Resuscitation 2018; 131:91-100.

29. Kim SJ, Kim HJ, Lee HY, Ahn HS, Lee SW. Comparing extracorporeal cardiopulmonary resuscitation with conventional cardiopulmonary resuscitation: a meta-analysis. Resuscitation 2016; 103:106-16.

30. Soar J, Maconochie I, Wyckoff MH, et al. 2019 international consensus on cardiopulmonary resuscitation and emergency cardiovascular care science with treatment recommendations. Resuscitation 2019; 145:95-150.

31. Bartos JA, Frascone RJ, Conterato M, et al. The Minnesota mobile extracorporeal cardiopulmonary resuscitation consortium for treatment of out-of-hospital refractory ventricular fibrillation: program description, performance, and outcomes. EClinicalMedicine 2020; 29-30:100632.

32. Richardson ASC, Tonna JE, Nanjayya V, et al. Extracorporeal cardiopulmonary resuscitation in adults. Interim guideline consensus statement from the Extracorporeal Life Support Organization. ASAIO J 2021; 67:221-8.

33. De Charrière $A$, Assouline $B$, Scheen $M$, et al. ECMO in cardiac arrest: a narrative review of the literature. J Clin Med 2021; 10:534.

34. Thiagarajan RR, Barbaro RP, Rycus PT, et al; ELSO member centers. Extracorporeal Life Support Organization registry international report 2016. ASAIO J 2017; 63:60-7.

35. Han SJ, Kim HS, Choi HH, et al. Predictors of survival following extracorporeal cardiopulmonary resuscitation in patients with acute myocardial infarctioncomplicated refractory cardiac arrest in the emergency department: a retrospective study. J Cardiothorac Surg 2015; 10:23.

36. Lee, JJ, Han SJ, Kim HS, et al. Out-of-hospital cardiac arrest patients treated with cardiopulmonary resuscitation using extracorporeal membrane oxygenation: focus on survival rate and neurologic outcome. Scand J Trauma Resusc Emerg Med 2016; 24:74.

37. Migdady I, Rice C, Deshpande A, et al. Brain injury and neurologic outcome in patients undergoing extracorporeal cardiopulmonary resuscitation: a systematic review and meta-analysis. Crit Care Med 2020; 48:e611-9. Erratum in: Crit Care Med 2020; 48:e845.

38. Kim SJ, Jung JS, Park JH, et al. An optimal transition time to extracorporeal cardiopulmonary resuscitation for predicting good neurological outcome in patients with out-of-hospital cardiac arrest: a propensity-matched study. Crit Care $2014 ; 18: 535$

39. Cardarelli M, Young A, Griffith B. Use of extracorporeal membrane oxygenation for adults in cardiac arrest (E-CPR): a meta-analysis of observational studies. ASAIO J 2009; 55:581-6.

40. Lamhaut L, Hutin A, Puymirat E, et al. A Pre-Hospital Extracorporeal Cardio Pulmonary Resuscitation (ECPR) strategy for treatment of refractory out hospital cardiac arrest: an observational study and propensity analysis. Resuscitation 2017; 117:109-17.

41. Aubin H, Petrov G, Dalyanoglu H, et al. A suprainstitutional network for remote extracorporeal life support: a retrospective cohort study. JACC Heart Fail 2016; 4:698-708

42. Harvey MJ, Gaies MG, Prosser LA. U.S. and international in-hospital costs of extracorporeal membrane oxygenation: a systematic review. Appl Health Econ Health Policy 2015; 13:341-57.

43. Matsuoka Y, Goto R, Atsumi T, et al; SAVE-J Study Group. Cost-effectiveness of extracorporeal cardiopulmonary resuscitation for out-of-hospital cardiac arrest: a multi-centre prospective cohort study. Resuscitation 2020; 157:32-8.

44. Dennis M, Zmudzki F, Burns B, et al; Sydney ECMO Research Interest Group. Cost effectiveness and quality of life analysis of extracorporeal cardiopulmonary resuscitation (ECPR) for refractory cardiac arrest. Resuscitation 2019; 139:49-56.

45. Kawashima T, Uehara H, Miyagi N, et al. Impact of first documented rhythm on cost-effectiveness of extracorporeal cardiopulmonary resuscitation. Resuscitation 2019; 140:74-80.

46. Barbaro RP, Odetola FO, Kidwell KM, et al. Association of hospital-level volume of extracorporeal membrane oxygenation cases and mortality. Analysis of the extracorporeal life support organization registry. Am J Respir Crit Care Med 2015; 191:894-901.

47. MacLaren G, Masoumi A, Brodie D. ECPR for out-of-hospital cardiac arrest: more evidence is needed. Crit Care 2020; 24:7. 